\title{
XLVII. Corpuscular radiation
}

\section{H. Bateman M.A. Ph.D.}

To cite this article: H. Bateman M.A. Ph.D. (1913) XLVII. Corpuscular radiation, Philosophical Magazine Series 6, 26:154, 579-585, DOI: 10.1080/14786441308635004

To link to this article: http://dx.doi.org/10.1080/14786441308635004

$$
\text { 册 Published online: } 08 \text { Apr } 2009 .
$$

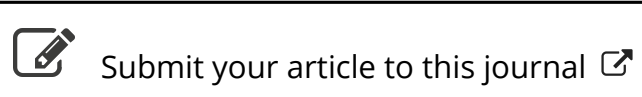

\footnotetext{
Џll Article views: 7
}

Q View related articles $\square$

4 Citing articles: 3 View citing articles $\square$ 
XLVII. Corpuscular Radiation. By H. Bateman, M.A., Ph.D., Teaching and Research Scholar, Johns Hopkins University *.

$\$ 1$. T $N$ the mathematical theory of electromagnetism it is customary to adopt the hypothesis that an electromagnetic field which occurs in nature can be obtained by superposing a number of elementary electromagnetic fields, each of which has a simple real point singularity moving through the æether with a velocity less than that of light. The solution of Maxwell's equations which corresponds to this elementary type of field has been given by Liénard $\dagger$ and Wiechert + it is found that a constant (positive or negative) charge is associated with the singularity, and on this account the elementary field is sometimes regarded as a model of that due to a moving electron: better results, however, have been obtained by regarding the electron as an aggregate of such point charges distributed throughout a small volume.

It is implicitly assumed that electromagnetic fields with other types of singularities are non-existent. This hy pothesis, however, is of such a sweeping nature that it ought only to be adopted after a careful study of the different types of singularities which solutions of Maxwell's equations can possess. After examining a few of these I have come across one type of electromagnetic field which may perhaps be of some physical interest as its chief characteristic is a corpuscular type of radiation. The brief description which has been given elsewhere $\S$ will now be amplified by a geometrical construction for the electric and magnetic vectors at an arbitrary point of space.

\$2. The primary singularity may be described as a double-barreled gun $\mathrm{G}$ which moves through the æther in an arbitrary manner and keeps up a perpetual fire of secondary singularities (bullets) \| which move with the speed of light along straight lines in the direction of the barrels of the gun. These directions vary with the velocity of $G$ in such a way that the angle between them is always bisected by the direction of G's motion and its magnitude depends on

* Communicated by the Author.

+ L'éclairage électrique, pp. 5, 53, 106 (1898).

\pm Archives nécrlandaises (2) T. p. 54 (1900).

$\$$ The Annals of Mathematics, Dec. 1913.

il As far as I can make out there is no total charge associated with either the gun or the bullets. 
the velocity of $G$, which is supposed to be less than the velocity of light (fig. 1).

Fig. 1

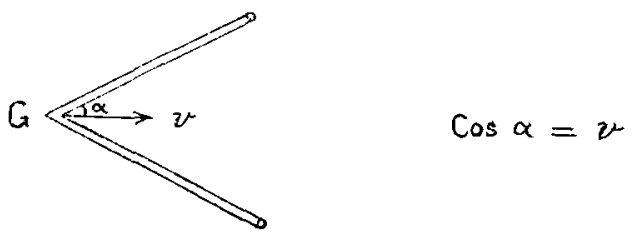

The electric and magnetic vectors at an arbitrary point $(x, y, z, t)$ can be calculated from the state of motion of the gun at one particular time $\tau$ (depending on $x, y, z, t$ ), the corresponding position of the gun may be called the effective position for the point $(x, y, z, t)$. When the barrels of the gun do not alter their direction the electric and magnetic vectors vanish; hence the electromagnetic field only comes into existence when the directions of the barrels change.

$\S 3$. Let the motion of the gun be represented by the equations

where

$$
x=\xi(\tau), \quad y=\eta(\tau), \quad z=\zeta(\tau),
$$

$$
\xi^{\prime 2}(\tau)+\eta^{\prime 2}(\tau)+\zeta^{\prime 2}(\tau)<1 .
$$

For each point $(x, y, z, t)$ in space there is then just one value of $\tau$ for which

$$
[x-\xi(\tau)]^{2}+[y-\eta(\tau)]^{2}+[\tilde{\tau}-\zeta(\tau)]^{2}=[t-\tau]^{2}, \quad t \geqslant \tau .
$$

(The unit of time is chosen so that the velocity of light is represented by unity.)

Now let functions $l(\tau), m(\tau), n(\tau)$ be chosen so that

then if

$$
\begin{aligned}
l^{2}+m^{2}+n^{2} & =1, \\
l \xi^{\prime}+m \eta^{\prime}+n \xi^{\prime} & =1,
\end{aligned}
$$

$$
w=l(x-\xi)+m(y-\eta)+n(z-\zeta)-(t-\tau),
$$

it is easy to verify that when $f(\tau)$ is an arbitrary function,

$$
\Omega=\frac{f(\tau)}{w}
$$

is a solution of the wave equation

$$
\frac{\partial^{2} \Omega}{\partial x^{2}}+\frac{\partial^{2} \Omega}{\partial y^{2}}+\frac{\partial^{2} \Omega}{\partial z^{2}}=\frac{\partial^{2} \Omega}{\partial t^{2}}
$$


The functions $l, m, n$ are always complex quantities, but we may obtain a real set of solutions of Maxwell's equations

by writing

$$
\operatorname{curl} \mathrm{E}=-\frac{\partial \mathrm{H}}{\partial t}, \quad \operatorname{curl} \mathrm{H}=\frac{\partial \mathrm{E}}{\partial t},
$$

$$
\mathrm{E}_{x}=-\frac{\partial \mathrm{A}_{x}}{\partial t}-\frac{\partial \Phi}{\partial x}, \quad \mathrm{H}_{x}=\frac{\partial \mathrm{A}_{z}}{\partial y}-\frac{\partial \mathrm{A}_{y}}{\partial z}
$$

where the potentials $A_{x}, A_{y}, A_{z}, \Phi$ are defined by tho equations

$$
\mathrm{A}_{x}=\mathbf{R} \frac{l}{w}, \quad \mathrm{~A}_{y}=\mathrm{R} \frac{m}{w}, \quad \mathrm{~A}_{z}=\mathrm{R} \frac{n}{w}, \quad \Phi=\mathrm{R} \frac{1}{w} ;
$$

the symbol $R$ being used to denote the real part of the quantity following it. These four potentials are solntions of the wave equation and satisfy the relation

$$
\frac{\partial A_{x}}{\partial x}+\frac{\partial A_{y}}{\partial y}+\frac{\partial A_{z}}{\partial z}+\frac{\partial \Phi}{\partial t}=0
$$

The components of the electric and magnetic vectors are found to be

where

$$
\begin{aligned}
\mathbf{E}_{x}= & \mathrm{R}\left\{-\frac{\partial \tau}{\partial t}\left(\frac{l^{\prime}}{w}-\mathrm{K} \frac{l}{w^{2}}\right)+\mathrm{K} \frac{\partial \tau}{\partial x} \cdot \frac{1}{w^{2}}\right\}, \\
& \ldots \ldots \ldots \ldots \ldots \ldots \ldots \ldots \ldots \ldots \ldots \ldots \ldots \ldots \\
\mathrm{H}_{x}= & \mathrm{R}\left\{\frac{\partial \tau}{\partial y}\left(\frac{n^{\prime}}{w}-\mathrm{K} \frac{n}{w^{2}}\right)-\frac{\partial \tau}{\partial z}\left(\frac{m^{\prime}}{w}-\mathrm{K} \frac{m}{w^{2}}\right)\right\},
\end{aligned}
$$

$$
\mathrm{K}=l^{\prime}(x-\xi)+m^{\prime}(y-\eta)+n^{\prime}(z-\zeta) \text {. }
$$

We easily see that

$$
\begin{aligned}
& \frac{\partial \tau}{\partial x} \mathrm{E}_{x}+\frac{\partial \tau}{\partial y} \mathrm{E}_{y}+\frac{\partial \tau}{\partial z} \mathrm{E}_{z}=0 \\
& \frac{\partial \tau}{\partial x} \mathrm{H}_{x}+\frac{\partial \tau}{\partial y} \mathrm{H}_{y}+\frac{\partial \tau}{\partial z} \mathrm{H}_{z}=0
\end{aligned}
$$

$x-\xi=\mathrm{M} \frac{\partial \tau}{\partial x}, \quad y-\eta=\mathrm{M} \frac{\partial \tau}{\partial y}, \quad z-\xi=\mathrm{M} \frac{\partial \tau}{\partial z}, t-\tau=-\mathrm{M} \frac{\partial \tau}{\partial t}$, where

$$
\mathrm{M}=\xi^{\prime}(x-\xi)+\eta^{\prime}(y-\eta)+\zeta^{\prime}(z-\zeta)-(t-\tau)
$$

hence it follows that Poynting's vector at any point is along the radius from the effective position of the gun. The directions of the electric and magnetic vectors are the same for all space time points which correspond to a given effective Phil. Mag. S. 6. Vol. 26. No. 154. Oct. 1913. $2 \mathrm{R}$ 
position of the gun and lie on a radius through this position. The magnitudes of the vectors, however, vary inversely as the distance from the gun, and so the energy per unit volume obeys the inverse-square law as far as points on the same radius are concerned. It may be verified after some laborious algebra that the electric and magnetic vectors are equal in magnitude and at right angles to one another *.

$\$ 4$. To obtain more convenient expressions for the components of the electric and magnetic vectors, we write

$$
x-\xi=\alpha(t-\tau), \quad y-\eta=\beta(t-\tau), \quad z-\zeta=\gamma(t-\tau),
$$

and regard $\alpha, \beta, \gamma$ as constants. We may then write

$$
\begin{aligned}
& \mathrm{E}_{x}=\mathrm{R} \frac{1}{\mathrm{M}} \frac{d}{d \tau} \frac{l-\alpha}{l a+m \beta+n \gamma-1} \\
& \ldots \ldots \ldots \ldots \ldots \ldots \ldots \ldots \ldots \ldots \\
& \mathrm{H}_{x}=\mathrm{R} \frac{1}{\mathrm{M}} \frac{d}{d \tau} \frac{n \beta-m \gamma}{l \alpha+m \beta+n \gamma-1} .
\end{aligned}
$$

Now let $(\lambda, \mu, v),\left(\lambda_{0}, \mu_{0}, \nu_{0}\right)$ be the direction-cosines of the barrels of the gun at time $\tau$. We then have

$$
\begin{gathered}
l \lambda+m \mu+n \nu=1, \quad l \lambda_{0}+m \mu_{0}+n v_{0}=1, \\
\mathrm{E}_{x}=\frac{1}{2 \mathrm{M}} \frac{d}{d \tau}\left[\frac{\lambda-\alpha}{\lambda \alpha+\mu \beta+\nu \gamma-1}+\frac{\lambda_{0}-\alpha}{\lambda_{0} \alpha+\mu_{0} \bar{\beta}+\nu_{0} \gamma-1}\right] \\
\cdots \cdots \cdots \cdots \cdots \cdots \cdots \cdots \cdots \cdots \cdots \cdots \cdots \cdots \cdots \cdots \cdots \cdots \\
\mathrm{H}_{x}=\frac{1}{2 \mathrm{M}} \frac{d}{d \tau}\left[\frac{\nu \beta-\mu \gamma}{\overline{\lambda \alpha}+\mu \beta+\nu \gamma-1}+\frac{\nu_{0} \beta-\mu_{0} \gamma}{\lambda_{0} \alpha+\mu_{0} \beta+\nu_{0} \gamma-1}\right] .
\end{gathered}
$$

To prove this we take the plane containing the two barrels of the gun at time $\tau$ as the plane $y=0$ and the tangent to G's path as axis of $x$. Then

$$
\begin{aligned}
& \xi^{\prime}=v, \quad \eta^{\prime}=0, \quad \zeta^{\prime}=0, \quad l=\frac{1}{v}, \quad m=i \sqrt{ }\left(\frac{1}{v^{2}}-1\right), n=0 ; \\
& \lambda=v, \quad \mu=0, \quad \nu=\sqrt{ }\left(1-v^{2}\right) ; \\
& \lambda_{0}=v, \quad \mu_{0}=0, \quad v_{0}=-\sqrt{ }\left(1-v^{2}\right) ; \\
& 2 \mathrm{R} \frac{l-\alpha}{l \alpha+m \beta+n \gamma-1}=\frac{1-v \alpha}{\alpha-v+i \beta \sqrt{\left(1-v^{2}\right)}}+\frac{1-v \alpha}{\alpha-v-i \beta \sqrt{ }\left(1-v^{2}\right)} \\
&=\frac{v-\alpha}{v \alpha-1+\gamma \sqrt{ }\left(1-v^{2}\right)}+\frac{v-\alpha}{v \alpha-1-\gamma \sqrt{ }\left(1-v^{2}\right)} \\
&=\frac{\lambda-\alpha}{\lambda \alpha+\mu \beta+v \gamma-1}+\frac{\lambda_{0}-\alpha}{\lambda_{0} \alpha+\mu_{0} \beta+v_{0} \gamma-1} .
\end{aligned}
$$

The other identities can be established in the same way.

* A geometrical proof of this result is given below, 
$\S 5$. Now let $Q, A, A_{0}$ be points on a unit sphere whose coordinates are $(\alpha, \beta, \gamma),(\lambda, \mu, \nu),\left(\lambda_{0}, \mu_{0}, \nu_{0}\right)$ respectively. Let $I I$ be the tangent plane at the point $T$ diametrically opposite to $\mathrm{Q}$ and let $\mathrm{Q} A, \mathrm{QA}_{0}$ meet $\Pi$ in the points $\mathrm{B}, \mathrm{B}_{0}$ respectively. Let $C$ be the middle point of $B B_{0}$, then if $\Gamma$ denote the vector CQ we have

$$
\mathrm{E}=\frac{1}{2 \mathrm{M}} \frac{d \Gamma}{d \tau} .
$$

The changes in the positions of $A$ and $A_{0}$ in time $d \tau$ must now be marked on the sphere and $d \Gamma$ is then represented by the change in the position of $\mathrm{C}$ (fig. 2).

Fig. 2.

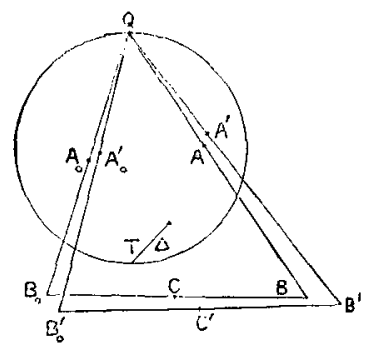

Again, if $\Delta$ represents a vector in the plane $\Pi$ at right angles to $\mathrm{TC}$ and equal in magnitude to $\mathrm{TC}$, we have

$$
H=\frac{1}{2 M} \frac{d \Delta}{d \tau} .
$$

If $d \Delta$ is rotated through a right angle in a suitable direction, it is also represented in magnitude and direction by the displacement of $\mathrm{C}$. This result proves at once that $\mathrm{E}$ and $H$ are perpendicular and equal in magnitude.

Since the factor $\frac{1}{2 \mathrm{M}}$ is always negative, the directions of the electric and magnetic vectors are indicated at once by our geometrical construction. It is evident that they are both perpendicular to the radius from the effective position of the gun.

$\S 6$. This last fact makes it possible for us to draw lines of electric and magnetic force on a sphere whose centre is G. It will be sufficient to take this as our unit sphere, for the lines of force on a larger sphere may be obtained by a simple magnification round G. 
It should first of all be noticed that the line $\mathrm{QC}$ meets the polar line of $\mathrm{AA}_{0}$ with regard to the sphere, for if $\mathrm{QC}$ meets the sphere again in $\mathrm{K}$, the four points $\mathrm{QRAA_{0 }}$ form an harmonic set on the circle QRA A.

If now we regard $A$ and $A_{0}$ as fixed, the correspondence between $Q$ and $R$ gives a conformal transformation of the sphere into itself, and the transformation is of such a nature that when we project stereographically on a plane from a fixed point $\mathrm{V}$ on the sphere and use the complex variable $z$ to denote the position of a point in the plane, the affixes $q, r, a, a_{0}$ of the projections of $\mathrm{Q}, \mathrm{R}, \mathrm{A}, \mathrm{A}_{0}$ are connected by a relation of the form *

$$
q r-\frac{1}{2}(q+r)\left(a+a_{0}\right)+\alpha a_{0}=0 .
$$

Again, if $\mathrm{A}_{0}^{\prime}, \mathrm{A}^{\prime}, \mathrm{C}^{\prime}$ denote the displaced positions of $A_{0}, A, C$ and $Q^{\prime}$ meets the sphere again in $S$, the affixes of the stereographic projections of $Q, A_{0}^{\prime}, A^{\prime}, S$ are connected by the relation

$$
q^{s-\frac{1}{2}(q+s)\left(a^{\prime}+a_{0}{ }^{\prime}\right)+a^{\prime} a_{0}{ }^{\prime}=0 .}
$$

Now the electric vector at $\mathrm{Q}$ lies in the plane $\mathrm{QCC}^{\prime}$ and so is in the direction of the tangent at $\mathrm{Q}$ to the circle $\mathrm{QRS}$ on the sphere. Using the same letters to denote the stereographic projections of the points $\mathrm{Q}, \mathrm{R}, \mathrm{S}, \mathrm{A}, \mathrm{A}_{0} \& \mathrm{c}$., we find that the stereographic projections of the lines of electric force are such that the line through $Q$ is tangent to the circle QRS. Let $q+d q$ denote the complex affix of a consecutive point on the line through $Q$, then, since the cross ratio of the affixes of four concyclic points is real, the cross ratio of $q, q+d q, r, s$ is real:

$$
\begin{gathered}
\therefore \quad d q\left[\frac{1}{q-r}-\frac{1}{q-s}\right] \text { is real, } \\
\text { i.e., } d q\left[\frac{q-\frac{1}{2}\left(a+a_{0}\right)}{(q-a)\left(q-a_{0}\right)}-\frac{q-\frac{1}{2}\left(a^{\prime}+a_{0}^{\prime}\right)}{\left(q-a^{\prime}\right)\left(q-a_{0}\right)}\right] \text { is real. }
\end{gathered}
$$

Hence, if

$$
\log \frac{(q-a)\left(q-a_{0}\right)}{\left(q-a^{\prime}\right)\left(q-a_{0}^{\prime}\right)}=\phi+i \psi
$$

the curves $\psi=$ constant are the projections of the electric lines of force, and it follows that the curves $\phi=$ constant are the projections of the magnetic lines of force. The curves

* This is an immediate consequence of the result given on p. 33 of Harkness and Morley's 'Introduction to the Theory of Analytic Functions' (London, 1898). 
$\phi=$ constant, $\psi=$ constant are evidently the level curves and stream-lines due to the two doublets $\mathrm{AA}^{\prime}, \mathrm{A}_{0} \mathrm{~A}_{0}^{\prime}$, where the flow takes place in two dimensions.

'J'o ind the points on the sphere for which the electric and magnetic vectors are zero we must find when $R$ and $S$ coincide. The line $\mathrm{QR}$ is then a common tractor of two pairs of polar lines *.

Putting $r=s$, we have two equations,

$$
\begin{aligned}
q r-\frac{1}{2}(q+r)\left(a+a_{0}\right)+a a_{0} & =0, \\
q r-\frac{1}{2}(q+r)\left(a^{\prime}+a_{0}{ }^{\prime}\right)+a^{\prime} a_{0}{ }^{\prime} & =0,
\end{aligned}
$$

which determine the values of $q$ and $r$ corresponding to the two real points cut out on the sphere by one of the tractors of the two pairs of polar lines. The other tractor is the polar line of the first with regard to the sphere and does not (generally) meet the sphere in real points.

Hence there are two real points on the sphere at which the electric and magnelic forces vanish. There are also two points at which the forces are infinite, viz. the points $\mathrm{A}$ and $\mathrm{A}_{0}$ in which the sphere is pierced by bullets.

It should be noticed that we have proved incidentally that two pairs of real polar lines with regard to a sphere always have two real tractors.

$\$ 7$. Let us now consider the reflexion of an electromagnetic disturbance of the type we have been studying when the mirror is an infinite plane and a perfect reflector. Let $x=0$ be the plane of the mirror, then we can satisfy the boundary condition at its surface by subtracting trom the original electromagnetic field another one of a similar type in which the motion of the gun is given by the equations

$$
x=-\xi(\sigma), \quad y=\eta(\sigma), \quad z=\zeta(\sigma),
$$

and the direction-cosines of its barrels are $-\lambda(\sigma), \mu(\sigma), \nu(\sigma)$; $-\lambda_{0}(\sigma), \mu_{0}(\sigma), \nu_{0}(\sigma)$ respectively.

When $(x, y, z, t)$ is on the mirror, $x=0$ and $\sigma=\tau$; the values of $\mathrm{E}_{y}, \mathrm{E}_{z}, \mathrm{H}_{x}$ are the same in both fields, and so when we subtract, the resultant electric force is normal to the mirror and the magnetic force tangential.

If this theory is correct the bullets strike the mirror and rebound in such a way that the angle of incidence is equal to the angle of reflexion, i. e. they are reflected like rays of light.

* I. e., a straight line which meets all four. The term is due to Cayley. 